\title{
Challenges and Progress in Low Defectivity for advanced ArF Lithography Processes using Surface Localized Material Technology
}

\author{
Michihiro Shirakawa ${ }^{1 *}$, Toru Fujimori ${ }^{1}$, Naohiro Tango ${ }^{1}$, Kazuhiro Marumo ${ }^{1}$, \\ Kei Yamamoto ${ }^{1}$, Hidenori Takahashi ${ }^{1}$, Ryo Nishio ${ }^{2}$, Akiyoshi Goto ${ }^{2}$, and \\ Mitsuhiro Fujita ${ }^{3}$
}

\author{
${ }^{I}$ Research \& Development management headquarters, Electronic Materials Research \\ Laboratories, FUJIFILM Corporation \\ ${ }^{2}$ Research \& Development management headquarters, Synthetic Organic Chemistry \\ Laboratories, FUJIFILM Corporation \\ ${ }^{3}$ Research \& Development management headquarters, Analysis Technology Center, \\ FUJIFILM Corporation \\ 1,2 4000 Kawashiri, Yoshida-cho, Haibara-gun, Shizuoka, 421-0396, Japan \\ TEL: +81-548-32-7312, FAX: +81-548-32-3185 \\ 3210 Nakanuma, Minamiashigara-shi, Kanagawa, 250-0193, Japan \\ TEL: +81-465-73-7080, FAX: +81-465-73-7923 \\ *michihiro.shirakawa@fujifilm.com
}

\begin{abstract}
The main challenge in ArF lithography is to reduce cost of ownership ( $\mathrm{CoO})$ because increase in multi-patterning process is generally required to obtain a fine pattern. As a consequence, industry strongly requires ArF lithography process with a fast scan speed scanner and low defectivity material for $\mathrm{CoO}$. The breakthrough technology to improve defectivity and resolution simultaneously was the polarity-change property of film surface from hydrophobic to hydrophilic after alkaline development process because a property after development process should be only associated with defectivity, not with fast scan speed. The materials with high polarity change function were explored to EUV process to achieve low defectivity with good lithography performances. Keywords: ArF immersion lithography extension, EUV lithography, Chemically amplified resist, Defectivity, Polarity change material
\end{abstract}

\section{Introduction}

Extreme ultraviolet lithography (EUVL) is one of the most promising candidates for high volume manufacturing of next generation lithography (NGL). On the other hand, ArF lithography process still occupies an important position for NGL due to the delay of EUVL by difficulties in developing exposure tools, masks, and resist materials. Materials for both EUV and ArF lithography are, therefore, necessary toward $10 \mathrm{~nm}$ node generation and beyond.

The main challenge in ArF lithography is to reduce cost of ownership $(\mathrm{CoO})$ because increase in multi-patterning process is generally required to obtain fine pattern [1]. As a consequence, industry strongly requires ArF lithography process with a fast scan speed scanner and low defectivity material for $\mathrm{CoO}$.

Top coat (TC) and TC-less resist are well-known as materials for $\mathrm{ArF}$ immersion exposure process (Fig.1) [2-5]. From the view point of material costs, TC-less resist process is more preferable than TC process.

We described herein novel polymer additive technology to satisfy both high scan speed and low defectivity requirements. The materials with high polarity change function were explored to EUV process to achieve low defectivity with good lithography performances. 

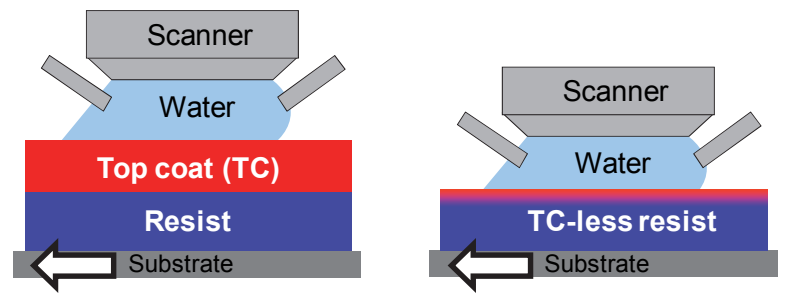

Fig. 1. Top coat process (left) and TC-less resist process (right).

\section{Experimental}

\subsection{Materials}

ArF resist compositions used in this study were prepared by dissolving methacrylate copolymer having an acid-decomposable group, photo acid generators (PAG), amines as quencher and hydrophobic polymer additives into organic solvents. The resulting solutions were filtered with $0.03 \mu \mathrm{m}$ polyethylene filter for lithography evaluations.

\subsection{Contact angle measurements}

The resist solution was spin-coated on silicon wafer and baked at $100{ }^{\circ} \mathrm{C}$ for $60 \mathrm{sec}$, and giving film thickness of $100 \mathrm{~nm}$. The resulting substrate was used for the contact angle measurements. The receding contact angle (RCA) of a water droplet was measured using a dynamic contact angle meter (manufactured by Kyowa Interface Science Co., Ltd.) by expansion-contraction method.

\subsection{Defect analysis}

Line and space patterns with $100 \mathrm{~nm}$ pitch 1:1 duty were printed on a 12-inch wafer and inspected. The defect distribution of the wafer was detected with UVision3+ (manufactured by AMAT) and the shape of the defects was observed using SEMcisionG4 (manufactured by AMAT).

\subsection{XPS measurement}

An XPS (X-ray Photoelectron Spectroscopy) measurement was used to measure the fluorine content at the surface of TC-less resists. The resist solution was spin-coated on silicon wafer and baked at $100{ }^{\circ} \mathrm{C}$ for $60 \mathrm{sec}$, and giving film thickness of $100 \mathrm{~nm}$. The resulting substrate was used for the XPS measurement.

\section{Results and discussion}

3.1. Demand to a TC-less resist

To improve followability at high scan speed immersion lithography, TC-less resist surface should have hydrophobic property, that is, higher RCA (receding contact angle) is required. The scan speed of the latest exposure tools rises in $800 \mathrm{~mm} / \mathrm{s}$ now [6]. We set more than 85 degree RCA as a target to keep good followability for these exposure tools. TC-less resists which have hydrophobic surface can be obtained by addition of hydrophobic polymers which can localize to resist surface. Polymer additives, which have various surface energy values, were added $1 \mathrm{wt} \%$ to a conventional ArF resist, then, SCA (static contact angle) values were measured in order to calculate surface coverage ratio. The result was shown in Fig. 2. This result indicates that polymer additives which have low surface energy are easy to localize to resist surface. Possible mechanism of this tendency is following, difference of surface energy between a resist polymer and a polymer additive promote phase separation of these polymers and a low surface energy polymer additive move to resist surface to minimize surface energy of a resist film. oxygen inhibition was effectively suppressed by the use of thiol-ene photocuring system due to regeneration of thiyl radical in the presence of oxygen [7] (Scheme 1). Thus, application of thiolene system may afford high resist sensitivity and durability. Effect of the structures of thiol compounds on flexibility of the cured resist and resist sensitivity was also investigated.

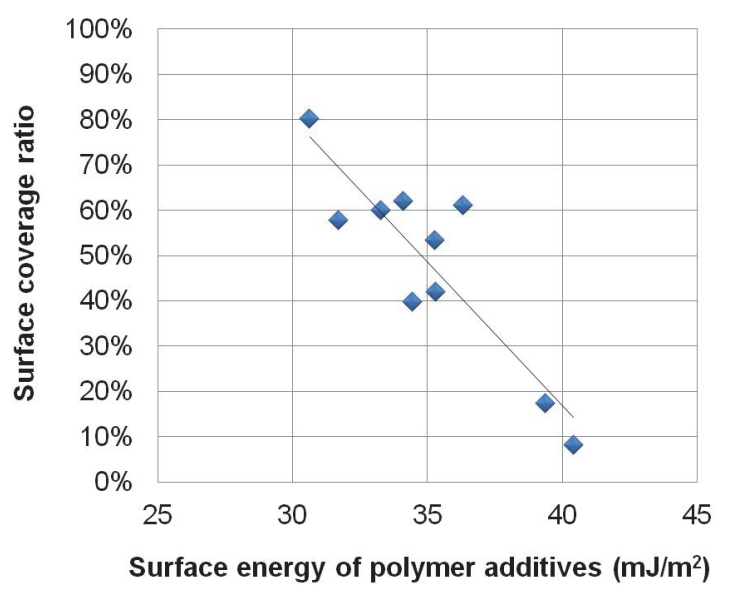

Fig. 2. Relationship between surface energy of polymer additives and surface coverage ratio.

Figure 3 shows wettabilities of water, alkaline developer for positive-tone development (PTD) process, and organic solvent developer for negativetone development (NTD) process [7], to high RCA hydrophobic resist surface. In the case 


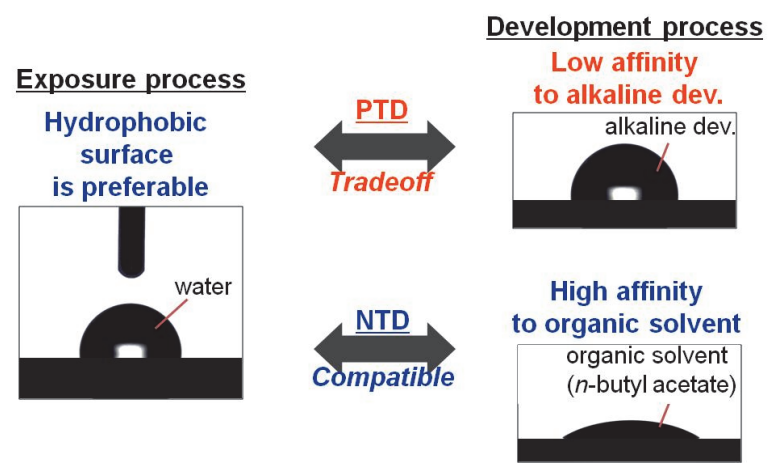

Fig. 3. Wettabilities of water (left), alkaline developer (right top), and organic solvent developer (right bottom) to a hydrophobic TC-less resist surface.

of NTD, organic solvent developer has high affinity to hydrophobic resist surface, indicating that fast scan speed and low defectivity, which should be affected by developer wettability, are compatible. On the other hand, in the case of PTD, fast scan speed and low defectivity might be a tradeoff relation because alkaline developer shows low affinity to hydrophobic resist surface.

We investigated the relationship between the number of defects and SCA after development, which describes wettability of resist surface to a developer and a rinse (Fig. 4). This result obviously indicates that the number of defects increases as SCA after development becomes high. It is necessary for a defect reduction that SCA after development should be 60 degree or less.

Summarizing the above, 85 degree or more RCA for fast scan speed and 60 degree or less SCA after development are required to a TC-less resist for the latest immersion exposure tools.

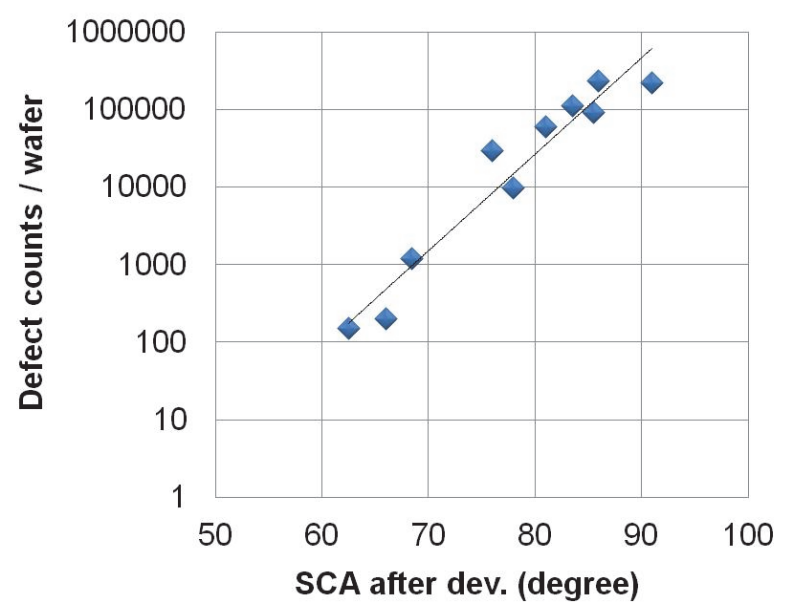

Fig. 4. Relationship between SCA values after development and the number of defects.

\subsection{Strategy of polarity change}

As shown in above section, a TC-less resist should have a hydrophobic property at an exposure step and a hydrophilic property at a development step. To meet this demand, a TC-less resist must have polarity change function. We considered three types of polarity change mechanism as shown in Fig. 5 . The first is an alkaline soluble polymer additive, which can be removed at a development step, the second is an acid-responsive polymer additive, which can be decomposed at an exposure step, and the third is an alkaline-responsive polymer additive, which can be decomposed at a development step.

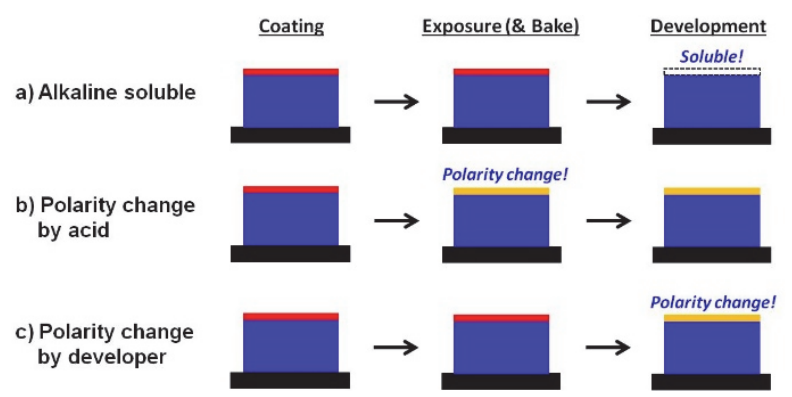

Fig. 5. Three types of polarity change mechanism.

Various polymer additives were synthesized by co-polymerization with hydrophobic units and alkaline-soluble units. Selected alkaline-soluble units are lactones, fluoro-alcohols, and sulfonimides. Figure 6 shows the relation of the ratio of alkalinesoluble units in co-polymer versus RCA. This result indicates that the introduction of all alkaline-soluble units makes RCA worse. We considered that introduction of alkaline-soluble units cannot keep high RCA because they must have highly

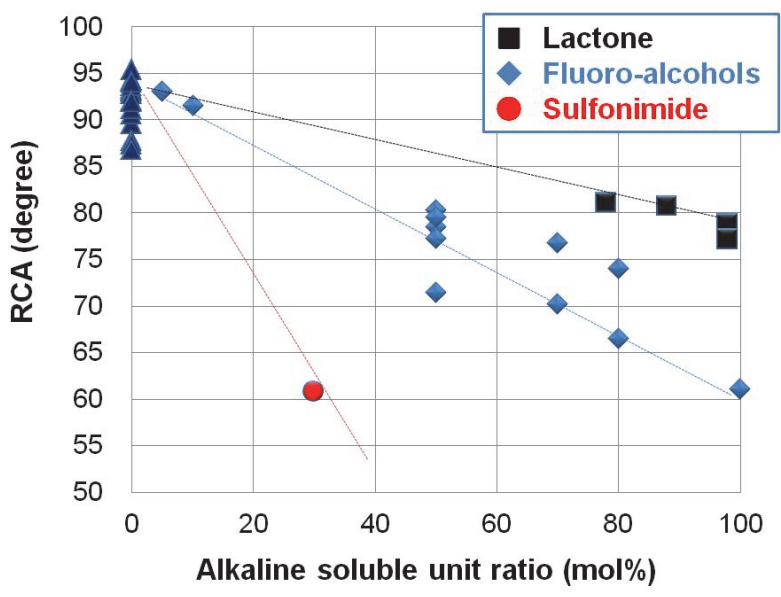

Fig. 6. Plots of alkaline soluble unit ratio versus RCA; Lactone (square), Fluoro-alcholos (diamond), Sulfoimide (circle) are used as alkaline soluble units. 


\section{Coating Exposure (\& Bake) Development}

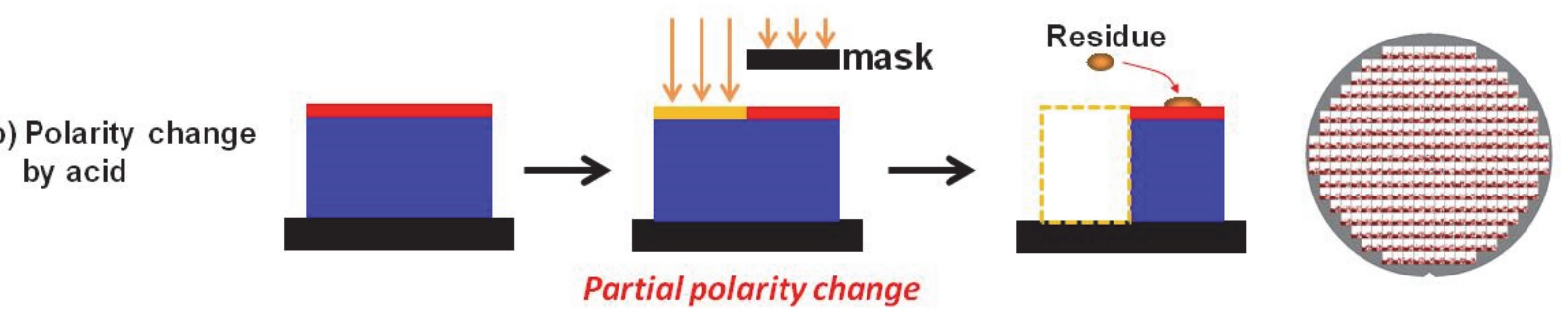

Fig. 7. Scheme and defect map of a TC-less resist using an acid-responsive polarity change polymer additive.

hydrophilic part in their units. We conclude that alkaline-soluble type never achieves high RCA and low SCA after development together.

Next, we studied a polymer additive which has an acid-responsive polarity change function. This type of polymer additive can change its polarity only at exposed region in principle. In order to investigate the risk of this character, we evaluated defect performance of the resist film which has large scaled unexposed region. As shown in Fig. 7, so many defects are observed at unexposed region. It is considered that residual components which adhere to resist surface cannot be removed because hydrophobic resist surface shed rinse water.

As both an alkaline soluble type and an acidresponsive type cannot achieve high RCA with low SCA after development, we investigated an alkaline-responsive type in detail.

\subsection{Investigation of an alkaline-responsive polymer} additive

We selected a linkage group which can be hydrolyzed by alkali-stimulus as an alkalineresponsive polarity change unit. Influence of spacer length between polymer backbone and the linkage group and an electron withdrawing strength of a terminal group on RCA and hydrolysis reactivity was studied as shown in Fig. 8. Longer space length is favorable for hydrolysis reactivity but unfavorable for RCA, and middle type space can balance RCA with alkaline reactivity. Furthermore, a strong electron withdrawing group (EWG) can accelerate hydrolysis reactivity drastically. From these results, we designed alkaline-responsive polymer additive-A which has middle spacer length with a strong EWG as the optimized structure.

Next, we evaluated a contact angle measurement of TC-less resists loading with various amount of alkaline-responsive polymer additive-A. Figure 9 suggests that addition of polymer additive-A can achieve 85 degree and more RCA and 60 degree and less SCA after development simultaneously. We measured surface fluorine content of the alkalineresponsive TC-less resist including polymer additive-A by XPS analysis to confirm the polarity change mechanism (Fig. 10). High fluorine content of the coated resist surface proves that additive-A has high surface localizable ability. Furthermore, the fact that fluorine cannot be detected after development shows the high polarity change ability of additive-A.

Comparison of the defect performance between a TC-less resist which contains a no polarity change polymer additive and a TC-less resist which contains an alkaline-responsive polymer additive was summarized in Table 1. As expected, a TCless resist with alkaline-responsive polymer additive can reduce defects drastically.
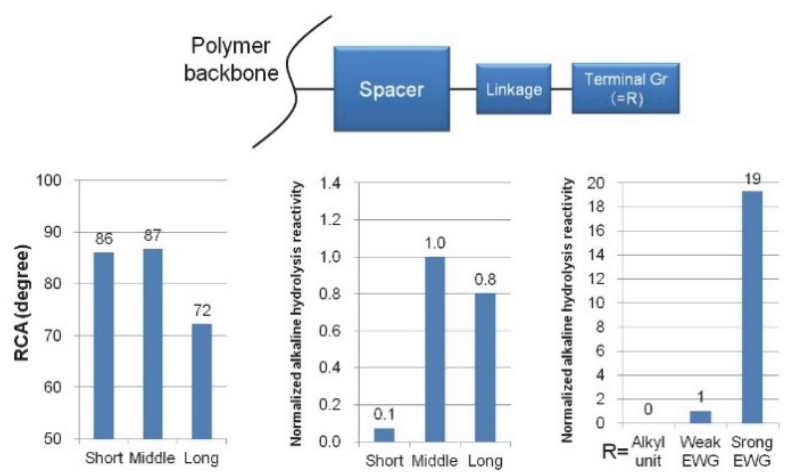

Fig. 8. Relationships between properties and designs of polymer additives; left) spacer length and RCA, center) spacer length and hydrolysis reactivity, and right) EWG strength and hydrolysis reactivity.

3.4. Effect of an alkaline-responsive polymer additive on a EUV resist

Polymer additive-A, which was developed for ArF immersion process as shown in the above section, was also evaluated for a EUV resist (Fig. 11). Although poly(4-hydroxystylene) (PHS) based EUV polymers have more hydrophobic nature compared to polymers for ArF, polymer additive-A shows high surface localizable and polarity change 
abilities as well as ArF case. This result indicates that the technology of alkaline-responsive polymer additive is useful for EUVL which is required more severe defect performance than ArF.

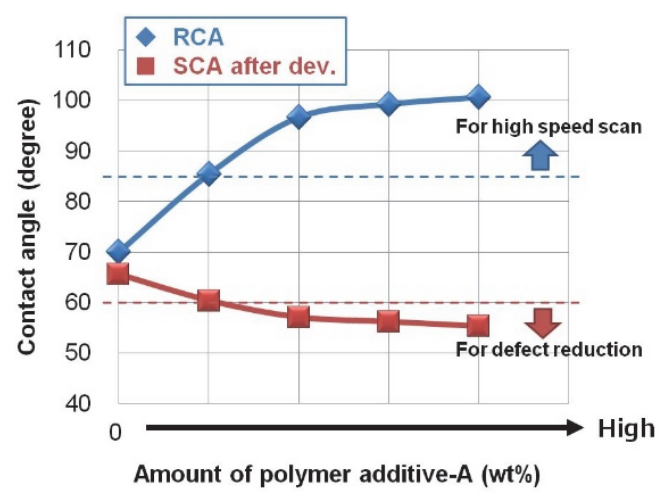

Fig. 9. Relationship between the amount of alkalineresponsive polymer additive-A and contact angle; (diamond) RCA after coating, (square) SCA after development.

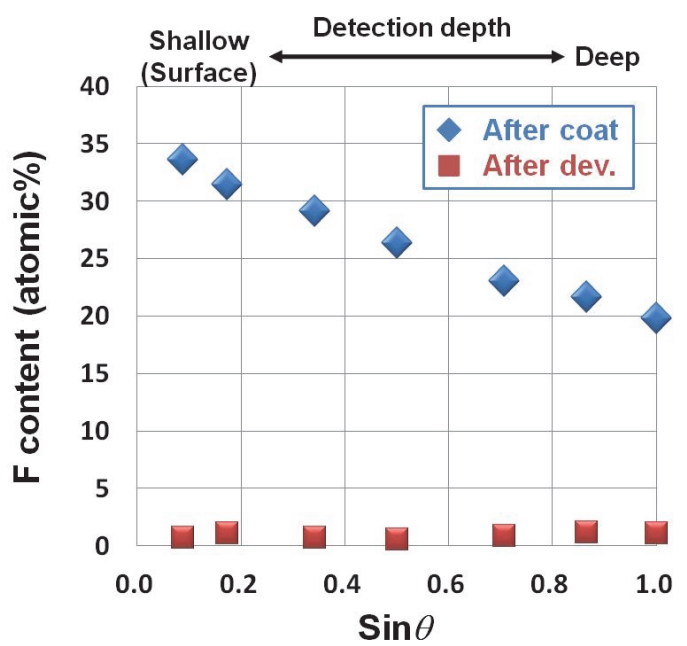

Fig. 10. Surface fluorine content of an alkalineresponsive TC-less resist detected by XPS analysis; (diamond) after coating, (square) after development.

Table 1. Comparison of the performance of high RCA TC-less resists which contain a no polarity change polymer additive and an alkaline-responsive polymer additive, respectively.

\begin{tabular}{|c|c|c|}
\hline Polymer additives & Hydrophobic type & $\begin{array}{c}\text { Alkaline } \\
\text { polarity change type }\end{array}$ \\
\hline Defect map & & \\
\hline
\end{tabular}

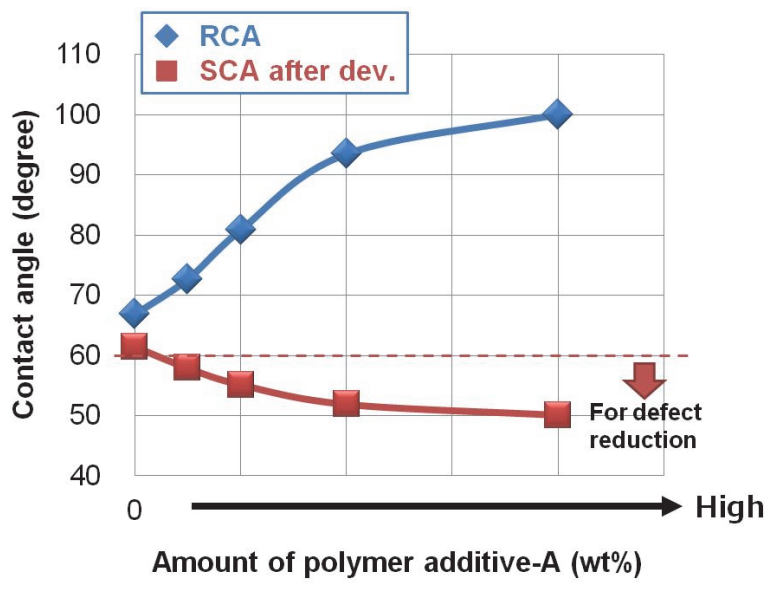

Fig. 11. Relationship between the amount of alkalineresponsive polymer additive-A to PHS based EUV polymer and contact angle; (diamond) RCA after coating, (square) SCA after development.

\section{Conclusion}

We explored three types of polymer additives for a next generation TC-less resist which has polarity change function to meet the demand for fast scan speed and low defectivity. A) Alkaline soluble, B) acid-responsive, and C) alkaline-responsive polymer additives were studied and we found that C) an alkaline-responsive polymer additive is the solution for this challenge. Suitable design of an alkaline-responsive unit enabled to achieve high RCA and low SCA after development simultaneously. This surface polarity change technology is expected to become a fundamental defect reduction method for not only ArF immersion lithography but also EUVL.

\section{References}

1. Y. Chen, Q. Cheng, and W. Kang, Proc. SPIE, 8326 (2012) 832620.

2. L. Marinier, Y. Aksenov, R. Morton, D. V. Streenwinckel, and P. Zandbergen, Proc. SPIE, 5753 (2005) 527.

3. W. D. Hinsberg, J. A. Hoffnagle, G. M. Wallraff; C. E. Larson, F. A. Houle, L. Sundberg, H. D. Truong, B. W. Davis, and R. D. Allen, Proc. SPIE, 5753 (2005) 508.

4. S. Kanna, S. Tarutani, N. Taguchi, T. Fukuhara, H. Kanda, K. Wada, K. Kodama, and K. Shitabatake, "Non topcoat resist design for immersion defectivity reduction", Presentation at the 3rd International Symposium on Immersion Lithography, Oct. (2006).

5. K. Nakano, R. Seki, T. Kawakubo, Y. Maruta, T. Sekito, K. Shiraishi, T. Sei, T. Fujiwara, T. 
Hayashi, Y. Iriuchijima, and S. Owa, Proc. SPIE, 7640 (2010) 76400X.

6. F. Bornebroek, "Extending ArFi immersion scanner capability in support of $1 x n m$ production nodes", Presentation at SPIE Advanced lithography. 27th Feb. (2014).

7. S. Tarutani, S. Kamimura, K. Fujii, K. Katou, and Y. Enomoto, Proc. SPIE, 7972 (2011) $79720 \mathrm{~N}$. 